\title{
EL RÉGIMEN DE ORTEGA. ¿UNA NUEVA DICTADURA FAMILIAR EN EL CONTINENTE? COORDINADO POR EDMUNDO JARQUÍN. MANAGUA: EDITORIAL PAVSA, 2016
}

El libro, tal y como lo señala el coordinador, tiene como propósito analizar la naturaleza del régimen de Ortega. De manera acertada, Jarquín señala en la presentación que Daniel Ortega "ha consolidado un poder personal y familiar, como nadie antes en la historia moderna de Nicaragua, incluido los Somoza. Ha constituido un régimen sultanístico, en que la voluntad e intereses del sultán se confunden con los del Estado" (8).

En esa línea, es un libro que, a lo largo de sus siete capítulos contribuye a desmitificar la narrativa oficial de la dupla Ortega-Murillo y del FSLN, que sostiene que son, por un lado, la continuidad de la Revolución Popular Sandinista y, segundo, que la legitimidad que tienen es democrática, resultado de un apoyo electoral mayoritario en los distintos procesos de votación que se han realizado entre 2006 y 2016, cuando fue publicado el libro. Por el contrario, este demuestra, desde distintos abordajes temáticos, por un lado, que en este período el gobierno actual no es una segunda parte de la Revolución o su continuidad y, por otro lado, demuestra que este gobierno no es la continuidad de la transición y consolidación democrática que se inició a partir de 1990. Lo que emerge con claridad de la lectura del libro es que Nicaragua ha vivido un proceso de ruptura con ambas líneas de construcción política.

Estamos frente a un Estado de distinta naturaleza que el revolucionario de los ochenta o el liberal-democrático del período 1990-2006. Si hubiera que compararlo con algún régimen de la historia previa de Nicaragua posiblemente se parecería al tipo de Estado que construyó la dinastía somocista. Como se analiza en varios de los capítulos, el carácter despótico del régimen se ha consolidado gracias al apoyo externo que le otorgó el padrinazgo político del ALBA y el apoyo cuantioso de la cooperación petrolera venezolana. E, internamente, gracias al acuerdo estratégico logrado con el sector empresarial nicaragüense, alianza que se terminó traduciendo en una reforma constitucional (artículo 98 de la Constitución Política), a la cooptación de una parte importante de los partidos políticos y a la exclusión de los que no se dejaron cooptar, 
y al acoso y la represión de las organizaciones de la sociedad civil y de los movimientos sociales críticos.

En el capítulo 1, Jarquín hace un tour de force analizando de manera resumida las principales transformaciones que ha vivido Nicaragua desde el pacto Ortega Alemán, pasando por el triunfo de Ortega en el 2006 y su continuidad hasta el 2016. Inicialmente discute qué tipo de régimen se ha instaurado en Nicaragua a partir del retorno de Ortega en 2006, concluyendo que es una suerte de repetición, en las circunstancias del siglo XXI, de los despotismos del pasado nicaragüense, confirmando el peso de la historia en el presente (ipath dependence?).

En un segundo momento, Jarquín analiza la relación de Ortega con el socialismo del siglo XXI y la incorporación de Nicaragua al ALBA, analizando las características compartidas por los países de ese espacio de integración regional. De ese análisis, queda claro que Nicaragua comparte aspectos centrales con los demás países (Bolivia, Ecuador, Venezuela), aunque no tiene una base amplia de apoyo o nueva mayoría y tiene características sultanísticas que los demás no tienen.

En un tercer momento, explica la forma en que Ortega obtuvo el poder dentro del FSLN. Particularmente relevante fue su estrategia después de la derrota electoral de 1990 y el cambio de régimen político que impulsó el gobierno de Violeta Barrios. En particular, la forma en que utilizó la organización de las protestas y la movilización social para consolidar su liderazgo (gobierno desde abajo), a la vez que conducía las negociaciones como contraparte del gobierno. También desarrolló un fuerte trabajo territorial y organizacional en todo el país en ese mismo período.

Otro hito en la consolidación de su liderazgo fue el pacto con el expresidente Alemán. Concediéndole inmunidad a este frente a los juicios por corrupción que tenía, no solo logró dividir al liberalismo sino también obtuvo importantes cuotas de poder por medio de la partidarización de los otros poderes del Estado y, más importante aún, cambios en la legislación electoral que, posteriormente, serían claves para permitir su reelección. Como parte del acuerdo, aprovechando las contradicciones de Alemán con el presidente Bolaños y con el líder liberal Eduardo Montealegre, logró despojar a este último de la personería jurídica de su partido, práctica que también ejerció contra la disidencia sandinista. En este mismo punto, Jarquín explica los fraudes electorales que se volvieron una constante en las elecciones que se desarrollaron durante su segundo período en particular el caso de las municipales de 2008.

De manera seguida, Jarquín analiza el apoyo venezolano en términos de renta externa. Durante el período 2008-14, el ingreso recibido implicó un monto promedio cercano a las USD 580 millones, para un total de cerca de USD 4059 millones, es decir, cerca del 6 por ciento del PIB. Entre los efectos derivados de este apoyo se puede mencionar que amplió el margen de maniobra con respecto a lo que Jarquín denomina condicionalidad democrática (la cooperación de EUA y europea, por ejemplo, exigen buenas prácticas de gobernanza). La segunda consecuencia fue la posibilidad 
de financiar programas sociales para ampliar la base de apoyo del gobierno de Ortega. La tercera consecuencia, pudo expandir gasto social a la vez que mantenía equilibrios financieros, validados por el FMI. Estos recursos le permitieron a Ortega consolidar la alianza con el sector empresarial estableciendo un nuevo consenso político al margen de los partidos políticos. Finalmente, la cooperación venezolana, al ser manejada de manera privada, le permitió a la familia Ortega-Murillo y a su círculo cercano, crear un poderoso grupo empresarial y articular buenos negocios con el sector empresarial. Entre los negocios de la familia se puede mencionar una compra grande de medios de comunicación.

Otro aspecto que analiza Jarquín es el relacionado con los fraudes electorales, en particular los de las elecciones municipales de 2008 y las presidenciales de 2006, así como la represión desatada por el régimen contra las protestas por ambos fraudes y el creciente partidismo de la Policía Nacional en el ejercicio de la coerción contra movimientos ciudadanos contra el gobierno, incluyendo el de \#OcupaINSS (2013).

En los siguientes apartados, Jarquín desarrolla otros temas claves para entender la consolidación del régimen sultanístico de Ortega: el manejo económico y los pactos con el sector empresarial, el acuerdo Ortega-EUA por la lucha anti-drogas y el tema de seguridad regional; su relación de cooperación con el cardenal Obando y Bravo; las modificaciones constitucionales para debilitar el Estado de derecho y subordinar la institucionalidad pública a su figura, avanzando en lo que Tünnermann denomina la "demolición de la República". También Jarquín menciona como parte de esa transformación constitucional la concesión canalera dada a un empresario aventurero hongkonés, lo que generó una fuerte movilización y protestas del campesinado afectado por las potenciales expropiaciones. El capítulo continúa con el análisis de la corrupción que ha caracterizado al régimen de Ortega, cuyos niveles se consideran más graves que los de Haití. En la parte final, analiza la situación de la equidad de género, sobre todo en términos de acceso a la educación y a la salud, desmitificando la imagen que ha creado el gobierno de Ortega Murillo de tener una política de género exitosa. No es casual que el movimiento feminista sea una de las principales fuerzas de oposición, sobre todo después de la denuncia que realizara en 1998, su hija adoptiva Zoilamérica acusándolo de haber sido abusada sexualmente por Ortega. Finalmente, el tour de force de Jarquín termina con el análisis de la subordinación partidaria y centralización, explicando cómo, por medio de instrumentos partidarios tales como los Consejos de Poder Ciudadano redujeron la participación ciudadana no partidaria y debilitaron la autonomía municipal. Los CPC fueron sustituidos posteriormente por los denominados "gabinetes de familia", que funcionan como instrumentos de control ciudadano y no de participación.

El capítulo dos, de Julio Icaza, parte de un análisis en que analiza las tensiones provocadas por la disputa existente alrededor de la orientación de la Constitución Política y, en consecuencia, la forma de organización del Estado. En esa línea, el autor desarrolló un recorrido de las reformas realizadas en distintos momentos de la historia 
reciente de Nicaragua: 1990-97 y las reformas constitucionales de 1995, reformas que incluyeron la no reelección.

En un segundo momento, analiza el denominado Pacto Alemán-Ortega y las reformas constitucionales del 2000, que tuvieron un gran alcance en varios ámbitos: el tema de la propiedad, reformas electorales que fueron claves para permitir que Ortega pudiera reelegirse más adelante, al bajar el porcentaje para ganar en primera ronda de $40 \%$ a $35 \%$ de los votos válidos, siempre que la diferencia mínima entre los candidatos que quedaran en primer y segundo lugar fuera no menor al 5 por ciento.

Más adelante analiza las reformas que se empiezan a desarrollar a partir del retorno de Ortega al poder en el 2006: la declaratoria de inconstitucional de la reelección presidencial, lo cual dio paso a que se pudiera reelegir de manera indefinida; el proceso por medio del cual el FSLN terminó controlando la Corte Suprema de Justicia y el Consejo Supremo Electoral; la leyes de defensa y seguridad que se aprobaron en varios momentos y que permitieron una repartidarización de la Policía Nacional y el Ejército, incluyendo la forma de elección de los jerarcas de ambas instituciones, que pasaron a depender de manera directa del presidente Ortega, generando una situación de lealtad personal de estas autoridades con él. A ello se agrega el análisis de las leyes del canal interoceánico, en la que el Estado nicaragüense aprobó una concesión en condiciones leoninas y de clara entrega de la soberanía nacional.

En síntesis, este capítulo hace un análisis exhaustivo de las principales reformas, transformaciones legales y constitucionales que se dieron en un período que va de mediados de los 90s al 2016, permitiendo analizar la ruta seguida por Ortega para configurar un entramado institucional y legal favorable a su régimen dinástico y que han terminado por subordinar la institucionalidad pública y sus autoridades a la voluntad y autoridad del presidente de la república, quien concentra un enorme poder para exigir el cumplimiento de su voluntad, en una suerte de hiperpresidencialismo.

En el Capítulo 3, el politólogo José Antonio Peraza analiza el proceso de transformación al que fue sometido el sistema electoral como consecuencia del Pacto OrtegaAlemán en el 2000, que llevó primero a la partidarización del Consejo Supremo Electoral (CSE) y, posteriormente, al control total del mismo por el partido oficial. Ello le permitió entre otras cosas, imponer un bipartidismo forzado por medio de la prohibición de los partidos que amenazaban el pacto o la hegemonía orteguista. Además, Peraza realiza un ejercicio de análisis de los resultados electorales de las elecciones de 2006, 2008 y 2011 (no se había realizado la de 2016, en las que Ortega llevó como candidata a vicepresidenta a Rosario Murillo, su esposa), en el que demuestra, más allá de las exclusiones partidarias, comportamientos electorales atípicos (el FSLN ganando en centros de votación en los que, históricamente, el liberalismo había ganado por gran diferencia). Un punto adicional es que, conforme avanzaba la consolidación autoritaria del control de Ortega y Murillo, la actitud hacia la participación de observadores electorales internos y externos se fue haciendo más hostil, hasta llegar a su prohibición. 
En el Capítulo 4, Elvira Cuadra analiza el proceso de transformación institucional que llevó a cabo Ortega a partir de 2006 con el Ejército y la Policía Nacional. El hilo conductor de las reformas constitucionales y las leyes aprobadas fue, primero, la cooptación por medio de prebendas y posteriormente, la subordinación directa y personal de los jerarcas de ambas entidades al presidente de la República. Para ello, en ambos casos, el presidente rompió el principio de no reelección de estos y la quitó a los órganos colegiados la designación de los respectivos jerarcas. De tal manera, que se generó una dinámica de dependencia directa de los jerarcas con el presidente, para garantizar su continuidad al frente de las instituciones. En este capítulo ya aparece claro que en el caso de la policía, esta empezará a operar no solo permitiendo sino coordinando con fuerzas paramilitares en varios momentos de represión que hubo a partir de 2007.

En el siguiente capítulo (5), Uriel Pineda hace un análisis exhaustivo de la forma en que el régimen dinástico fue utilizando de manera cada vez más clara el poder despótico del sistema para reprimir las distintas formas de protesta que fueron desarrollándose a lo largo de esos años, incluyendo la represión de las protestas campesinas contra el canal y las relacionadas con Ocupa INSS, en la que se reprimió con grandes niveles de violencia a un grupo de jóvenes que se solidarizaron con adultos mayores que reclamaban su pensión. Es importante destacar, como ya se mencionó en la referencia al capítulo anterior, que la Policía permitió que los grupos de la Juventud Sandinista y de grupos vandálicos fueran los que llevaran a cabo la agresión a las personas que protestaban y el robo y destrucción de pertenencias variadas. Otra modalidad de operación también se empezó a desarrollar desde el inicio mismo del retorno de Ortega, cual fue el asfixiamiento de las ONG por medio de la interrupción de los flujos financieros de la cooperación internacional y el acoso legal por parte del Estado.

El capítulo 6 analiza la situación de la libertad de expresión. En este caso, Rotshchuh describe dos patrones de operación del régimen Ortega-Murillo: por un lado, mantiene una situación de acoso a los medios independentes o críticos. Por otro lado, la familia gobernante ha adquirido una gran cantidad de medios de comunicación gracias al manejo privado de la copiosa y generosa cooperación venezolana. Ello le ha permitido al gobierno tener un fuerte control de la agenda mediática del país y una importante posición de ventaja con respecto a gobiernos anteriores e incluso, con respecto a los demás países del ALBA, que no tienen esa ventajosa posición.

El capítulo 7 y final, Enrique Sáenz hace un balance del modelo económico y su gestión por parte del gobierno de Ortega. En este, lo primero que destaca son las condiciones favorables que tuvo Ortega desde el inicio y durante buena parte de su gestión. Primero, recibió una herencia del gobierno anterior de bajo endeudamiento, baja inflación, con crecimiento económico y una Nicaragua reinsertada en el comercio internacional, y con una coyuntura favorable en términos de los precios de las materias primas que el país exportaba. Segundo, iniciando su primer período 
inició la transferencia de recursos de la copiosa cooperación venezolana (más de USD 4400 millones) y a ello debe agregarse un importante flujo de recursos de la cooperación internacional (aunque esta fue decreciendo conforme aumentó el perfil autoritario del estilo de gobierno de Ortega) y el financiamiento de la banca multilateral, en particular del BM, el BID y el BCIE. En este apartado, al igual que en el de Jarquín, se describe la alianza estratégica del régimen de Ortega con el sector empresarial, que incluso se consolidó de tal manera que el FSLN modificó la Constitución Política para incluirla. Obviamente, el entorno económico favorable, sumado al espacio en la toma de decisiones económicas que le otorgó Ortega a la elite empresarial, le permitió pedir a cambio un silencio cómplice sobre el devenir autoritario del régimen, incluyendo los fraudes electorales y el creciente uso de la fuerza para reprimir a la oposición política y ciudadana. Sin embargo, el artículo de Sáenz no solo describe la alianza sino que, con datos oficiales, demuestra, por un lado, el éxito relativo del manejo económico del régimen. Para ponerlo en sus términos, con el flujo financiero cuantioso que recibió Ortega en el período estudiado, el crecimiento económico es más bien modesto e, incluso, inferior al crecimiento promedio obtenido en los gobiernos anteriores. Por otro lado, también demuestra el fracaso de las políticas sociales de un gobierno que se autodenomina de los pobres.

En síntesis, el libro hace una completa radiografía crítica de la transformación sociopolítica de Nicaragua durante el período 2006-2016. Al final de su lectura, la tesis inicial de Jarquín, de que estábamos frente a un régimen dinástico y sultanístico se confirma. Sin embargo, hay algunos temas que están tratados de manera periférica o están ausentes. Por ejemplo, el papel crecientemente protagónico de Rosario Murillo, el tema del papel de las iglesias y de los movimientos sociales y las luchas ciudadanas; la variable geopolítica, en particular la permisividad del gobierno de Estados Unidos con esta transformación autoritaria, entre otros temas.

Termino señalando que, aunque el libro tiene tres años de haber sido publicado, resulta de referencia obligatoria para comprender la coyuntura que se inició en Nicaragua a partir de abril de 2018 y entender que la misma se venía tejiendo desde antes de la llegada de Ortega y Murillo al poder.

Alberto Cortés Ramos. Costarricense. Tiene un Doctorado en Geografía de la Universidad de Loughborough, Inglaterra; una Maestría en Estudios del Desarrollo, que obtuvo en el Instituto de Estudios Sociales (ISS) en Holanda; y es licenciado en Ciencias Políticas de la Universidad de Costa Rica (UCR). Profesor catedrático de la Escuela de Ciencias Políticas de la UCR.

Contacto: alberto.cortes@ucr.ac.cr

ORCID: 0000-0001-7038-8301 